\title{
Fast Reversed-Phase Liquid Chromatography to Reduce Back Exchange and Increase Throughput in H/D Exchange Monitored by FT-ICR Mass Spectrometry
}

\author{
Hui-Min Zhang, ${ }^{\text {a,b }}$ George M. Bou-Assaf, ${ }^{a, c}$ Mark R. Emmett, ${ }^{\text {a,c }}$ and \\ Alan G. Marshall ${ }^{\mathrm{a}, \mathrm{c}}$ \\ a National High Magnetic Field Laboratory, Florida State University, Tallahassee, Florida, USA \\ ${ }^{\mathrm{b}}$ Institute of Molecular Biophysics, Florida State University, Tallahassee, Florida, USA \\ ${ }^{\mathrm{c}}$ Department of Chemistry and Biochemistry, Florida State University, Tallahassee, Florida, USA
}

In solution-phase hydrogen/deuterium exchange (HDX), it is essential to minimize the back-exchange level of $\mathrm{H}$ for $\mathrm{D}$ after the exchange has been quenched, to accurately assign protein conformation and protein-protein or protein-ligand interactions. Reversed-phase HPLC is conducted at low $\mathrm{pH}$ and low temperature to desalt and separate proteolytic fragments. However, back exchange averages roughly $30 \%$ because of the long exposure to $\mathrm{H}_{2} \mathrm{O}$ in the mobile phase. In this report, we first show that there is no significant backbone amide hydrogen back exchange during quench and digestion; backbone exchange occurs primarily during subsequent liquid chromatography separation. We then show that a rapid reversed-phase separation reduces back exchange for HDX by at least $25 \%$, resulting from the dramatically reduced retention time of the peptide fragments on the column. The influence of retention time on back exchange was also evaluated. The rapid separation coupled with high-resolution FT-ICR MS at $14.5 \mathrm{~T}$ provides high amino acid sequence coverage, high sample throughput, and high reproducibility and reliability. (J Am Soc Mass Spectrom 2009, 20, 520-524) (C 2009 Published by Elsevier Inc. on behalf of American Society for Mass Spectrometry

S olution-phase hydrogen/deuterium exchange (HDX) coupled with mass spectrometry is a non-perturbative method to study protein conformational changes in protein-ligand and protein-protein interactions [1, 2]. The exchange reaction rate minimizes at $\mathrm{pH}$ 2.0-3.0 and at a temperature of about $0{ }^{\circ} \mathrm{C}$. To minimize back exchange of amide backbone -ND to $-\mathrm{NH}$, the procedures after quench are performed under quench conditions. However, back exchange still averages nearly 30\% [3], depending on various factors, e.g., peptide sequence [4]. With matrix-assisted laser desorption/ionization, the back-exchange level is even higher [5].

In addition to low $\mathrm{pH}$ and low temperature, the $\mathrm{H}_{2} \mathrm{O}$ concentration should be kept low and exposure to $\mathrm{H}_{2} \mathrm{O}$ should be minimized during and after quench. Thus, elution of peptide fragments should occur as fast as possible. Our standard reversedphase liquid chromatography (RPLC) $\mathrm{C}_{5}$ column

Address reprint requests to Dr. Alan G. Marshall, Florida State University, National High Magnetic Field Laboratory, 1800 E. Paul Dirac Drive, Tallahassee, FL 32310-4005. E-mail: marshall@magnet.fsu.edu elutes peptide fragments in about $4.5 \mathrm{~min}$. Supercritical fluid chromatography (SFC) reduces back exchange because its mobile phase is mainly $\mathrm{CO}_{2}$ instead of $\mathrm{H}_{2} \mathrm{O}$ [6]. Dramatic reduction of back exchange has been achieved with SFC separation. However, short column life and reliability of the experiment still require improvement.

Here we present results with a high flow rate reversedphase ProZap ${ }^{\mathrm{TM}} \mathrm{C}_{18}$ column to reduce back exchange, while also increasing sequence coverage and total fragment number. Compared to a conventional $\mathrm{C}_{5}$ column, the ProZap $\mathrm{C}_{18}$ column has a wider inside diameter, shorter length, smaller particle size, and larger pore size, enabling higher flow rate and lower back pressure. The retention time is thereby reduced from $4.5 \mathrm{~min}$ (Jupiter ${ }^{\mathrm{TM}} \mathrm{C}_{5}$ column) to $1.5 \mathrm{~min}$, for a 3-fold reduction in $\mathrm{H}_{2} \mathrm{O}$ exposure time and concomitant reduction in back exchange for the fragment peptides. Besides reduction in back exchange, the ProZap column also provides much improved elution efficiency for increased fragment number and amino acid sequence coverage. The fast elution of many peptide fragments results in increased mass spectral complexity, which greatly benefits from a high-resolution (high peak ca-
(C) 2009 Published by Elsevier Inc. on behalf of American Society for Mass Spectrometry. 1044-0305/09/\$32.00

doi:10.1016/j.jasms.2008.11.010
Published online November 21, 2008 Received July 8, 2008 Revised November 15, 2008 Accepted November 17, 2008 
pacity) $14.5 \mathrm{~T}$ Fourier transform ion cyclotron resonance (FT-ICR) mass spectrometer [7].

\section{Experimental}

\section{Materials}

Myoglobin from equine heart, protease type XIII from Aspergillus saitoi, deuterium oxide, formic acid, and luteinizing hormone releasing hormone (LHRH) were purchased from Sigma-Aldrich (St. Louis, MO, USA).

\section{Hydrogen/Deuterium Exchange}

The entire HDX experiment was automated with a LEAP robot (HTS PAL, Leap Technologies, Carrboro, NC, USA) [8]. A $5 \mu \mathrm{L}$ stock protein solution $(80 \mu \mathrm{M}$ for myoglobin) was mixed with $45 \mu \mathrm{L} 50 \mathrm{mM}$ sodium phosphate buffer in $\mathrm{D}_{2} \mathrm{O}(\mathrm{pH}$ meter reading $=7.8)$ to initiate each $\mathrm{H} / \mathrm{D}$ exchange period at about $0{ }^{\circ} \mathrm{C}$. For the $56 \mathrm{~h}$ exchange experiments (at $\left.25^{\circ} \mathrm{C}\right), \mathrm{LHRH}(5 \mu \mathrm{M})$ was incubated in $99.5 \% \mathrm{D}_{2} \mathrm{O}$; myoglobin $(8 \mu \mathrm{M})$ in 95\% deuterated buffer; and KIT (a receptor tyrosine kinase at $8 \mu \mathrm{M})$ in $92 \% \mathrm{D}_{2} \mathrm{O}$ buffer. Each $\mathrm{H} / \mathrm{D}-$ exchanged sample is then subjected to simultaneous quench and proteolysis [9].

\section{On-line LC/SFC ESI FT-ICR MS}

A Jasco HPLC/SFC instrument (Jasco, Easton, MD, USA) was interfaced to the LEAP robot. For liquid chromatography (LC), the protein digest was injected from a $30 \mu \mathrm{L}$ loop to either a $\mathrm{C}_{5}$ column (Jupiter ${ }^{\mathrm{TM}}$, Phenomenex, Torrance, CA, USA; $1 \times 50 \mathrm{~mm}, 5 \mu \mathrm{m}$ particle size, 300 Å pore size) or a ProZap ${ }^{\mathrm{TM}} \mathrm{C}_{18}$ column (Grace Davidson, Deerfield, IL, USA; HR, $1.5 \mu \mathrm{m}, 500$ $\AA$, $2.1 \times 10 \mathrm{~mm})$. The gradient is $2 \% \mathrm{~B}$ to $95 \% \mathrm{~B}$ in 1.5 min (short) or $15 \mathrm{~min}$ (long) (A: acetonitrile $/ \mathrm{H}_{2} \mathrm{O} /$ formic acid, 5/94.5/0.5; B: acetonitrile $/ \mathrm{H}_{2} \mathrm{O} /$ formic acid, $95 / 4.5 / 0.5)$, at $50 \mu \mathrm{L} / \mathrm{min}\left(\mathrm{C}_{5}\right.$ column $)$ or 0.3 $\mathrm{mL} / \mathrm{min}\left(\mathrm{C}_{18}\right.$ column).

SFC was conducted with a $4.6 \times 50 \mathrm{~mm}$ Atlantis $^{\mathrm{TM}}$ HILIC 5- $\mu \mathrm{m}$ silica column (Waters Corporation, Milford, MA, USA), with a 4-min gradient from 80 to $40 \%$ $\mathrm{CO}_{2}$ at $3.5 \mathrm{~mL} / \mathrm{min}$. The mobile phase modifier was acetonitrile/methanol $/ \mathrm{H}_{2} \mathrm{O} /$ formic acid, 40/40/19/1. The ionized eluent was directed to a custom-built hybrid LTQ (Thermo Electron Corp., San Jose, CA, USA) 14.5 T FT-ICR mass spectrometer. Mass spectra were collected from $400<\mathrm{m} / z<2000$ at high mass resolving power $\left(\mathrm{m} / \Delta \mathrm{m}_{50 \%}=200,000\right.$ at $\left.\mathrm{m} / \mathrm{z} 400\right)$. High magnetic field [10] provided excellent external calibration mass accuracy (typically better than 500 ppb rms).

\section{Data Analysis}

Data were collected with Xcalibur software (ThermoFisher, San Jose, CA, USA) and analyzed by an in-house analysis package [11], permitting reliable identification

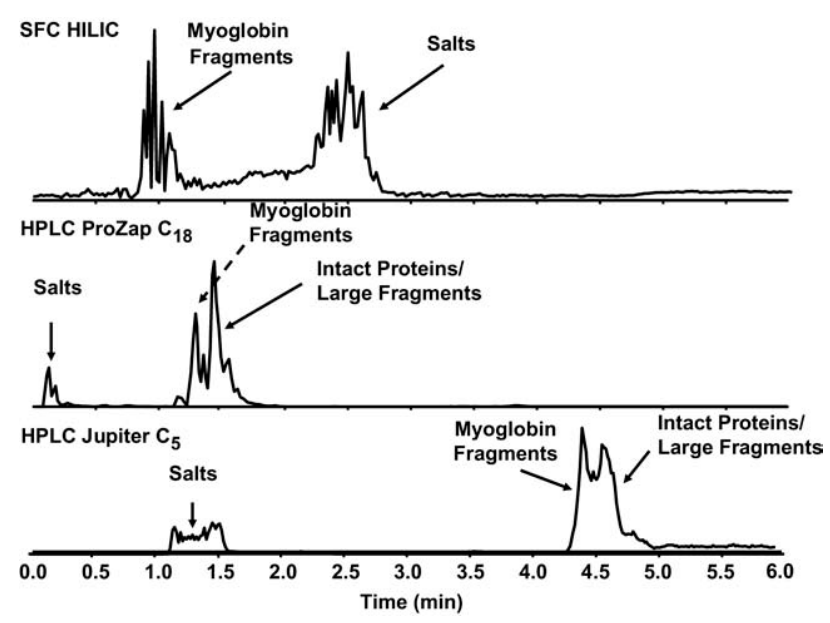

Figure 1. Elution profiles for myoglobin fragment peptides through an SFC Waters HILIC column (top), HPLC ProZap ${ }^{\mathrm{TM}} \mathrm{C}_{18}$ column (middle), and HPLC Jupiter ${ }^{\mathrm{TM}} \mathrm{C}_{5}$ column (bottom), with respective retention times of approximately $0.5-1.2 \mathrm{~min}, 0.7-1.5$ $\mathrm{min}$, and 4.0-4.5 $\mathrm{min}$.

and accurate assignment of the deuterium incorporation in each of the peptide fragments in the spectrum.

\section{Results and Discussion}

\section{Reduced Retention Time}

Figure 1 shows the total ion chromatogram (TIC) of the peptic fragments of myoglobin from HPLC Jupiter $\mathrm{C}_{5}$, HPLC ProZap $\mathrm{C}_{18}$, or SFC Waters HILIC separations. With the 1.5-min short gradient, the ProZap $\mathrm{C}_{18}$ column eluted peptic fragments from about 0.7 to $1.5 \mathrm{~min}-$ three times faster than the Jupiter $C_{5}$ column $(\sim 4.0-4.5$ min). The fast elution with the ProZap $\mathrm{C}_{18}$ column results from its wide i.d., short length, and large pore size, permitting high flow rate $(0.3 \mathrm{~mL} / \mathrm{min}$ versus 50 $\mu \mathrm{L} / \mathrm{min}$ for the $\mathrm{C}_{5}$ column) at low pressure ( $\left.10 \mathrm{MPa}\right)$. The small particle size and large pore size permit ultrafast and high-resolution peptide separations. In addition, the loading period was reduced to $0.5 \mathrm{~min}$ (versus $2 \mathrm{~min}$ for the $\mathrm{C}_{5}$ column). All of these factors contribute to faster elution of the peptides, less exposure to $\mathrm{H}_{2} \mathrm{O}$, and less back-exchange during LC separation for HDX MS analysis.

The fast separation also reduces the experiment duration and memory storage for more efficient data reduction and potentially higher throughput (more LC separation runs for the same experiment duration). Furthermore, the short retention time also allows more time for the column to fully re-equilibrate for improved reproducibility.

For all three separation methods with a short gradient, most of the fragment peptides eluted in a 30-s window. The complex mixture of co-eluted fragments is nevertheless resolved with our 14.5 T FT-ICR mass spectrometer, at a routine resolving power of $\mathrm{m} / \Delta \mathrm{m}_{50 \%}=$ 200,000 at $\mathrm{m} / \mathrm{z} 400$ for online LC operation. Charge 
states and monoisotopic $m / z$ values are accurately resolved with an in-house analysis package [11].

\section{Higher Elution Capacity}

The ProZap $\mathrm{C}_{18}$ column demonstrated a high capability to elute and separate many peptides, with shorter peptide average length $(\sim 17$ aa) than the SFC HILIC column ( $\sim 23 \mathrm{aa})$. The Jupiter $C_{5}$ column retained the shortest peptides ( $\sim 15 \mathrm{aa})$. The peptides eluted from the SFC HILIC column are relatively large because small hydrophilic peptides are not well solubilized by supercritical $\mathrm{CO}_{2}$. Because of charge competition in the electrospray ionization (ESI) process, larger fragments are ionized more efficiently. The ProZap $\mathrm{C}_{18}$ column yielded the most overlapping fragments (77 versus 48 for the $\mathrm{C}_{5}$ column and 25 for the SFC HILIC column) and the highest sequence coverage $(98.7 \%$ versus $96.8 \%$ for the $\mathrm{C}_{5}$ column and $93.5 \%$ for the SFC HILIC column), enabling amino acid sequence resolution of HDX rates to a few backbone amide hydrogens (sometimes even one or two).

\section{Reduced Back Exchange}

Figure 2 illustrates deuterium incorporation after 240 and $900 \mathrm{~s}$ exchange periods for each of four myoglobin fragments, following each of three different separations. Deuterium incorporation for the SFC HILIC is higher than that for ProZap $C_{18}$, which is higher than that for Jupiter $C_{5}$. The order of ability to reduce back exchange is thus the same.
Backbone Amide Hydrogen Back Exchange Occurs Primarily during LC Separation, Not during Quench and Proteolysis

The deuterium incorporation for fully exchanged LHRH was used to determine the absolute backexchange level, by comparing direct infusion and reversed-phase separation. LHRH, pyroGlu-His-TrpSer-Tyr-Gly-Leu-Arg-Pro-Gly-CONH ${ }_{2}$, has 10 amino acids. The number of potentially exchangeable backbone amide hydrogens is thus 9 (minus one for proline and plus one for the pyroGlu N-terminus), for a total of 9 backbone amide hydrogens and a grand total of 19 potentially exchangeable hydrogens (including side chains). Deuteration and back-exchange level were determined in three ways. First, direct infusion microelectrospray from the deuterated buffer was performed after addition of $100 \%$ formic acid (protic, final concentration in the solution is $0.5 \%$ ). Second, fully exchanged LHRH solution was injected onto a ProZap $\mathrm{C}_{18}$ column (1.5-min gradient). Third, fully exchanged LHRH was injected onto a Jupiter $C_{5}$ column (1.5-min gradient). Back exchange during a 2-min sham digestion procedure was simulated by adding $1 \%$ formic acid (in $\mathrm{H}_{2} \mathrm{O}$, no protease present) at a ratio of $1: 1(\mathrm{vol} / \mathrm{vol})$ into the LHRH solution at $0{ }^{\circ} \mathrm{C}$, analyzed by direct infusion.

Figure 3 shows ESI FT-ICR mass spectra of LHRH with the direct infusion after 56-h deuterium exchange (Figure 3b) and after 2-min sham digestion (Figure 3c). Figure $3 \mathrm{a}$ resulted from direct infusion of $\mathrm{LHRH}$ in $\mathrm{H}_{2} \mathrm{O}$. In Figure 3b, LHRH incorporated close to 17 deuteriums (out of a possible 19), indicating that the LHRH backbone amide hydrogens were fully exchanged and two of the exchanged deuteriums on the

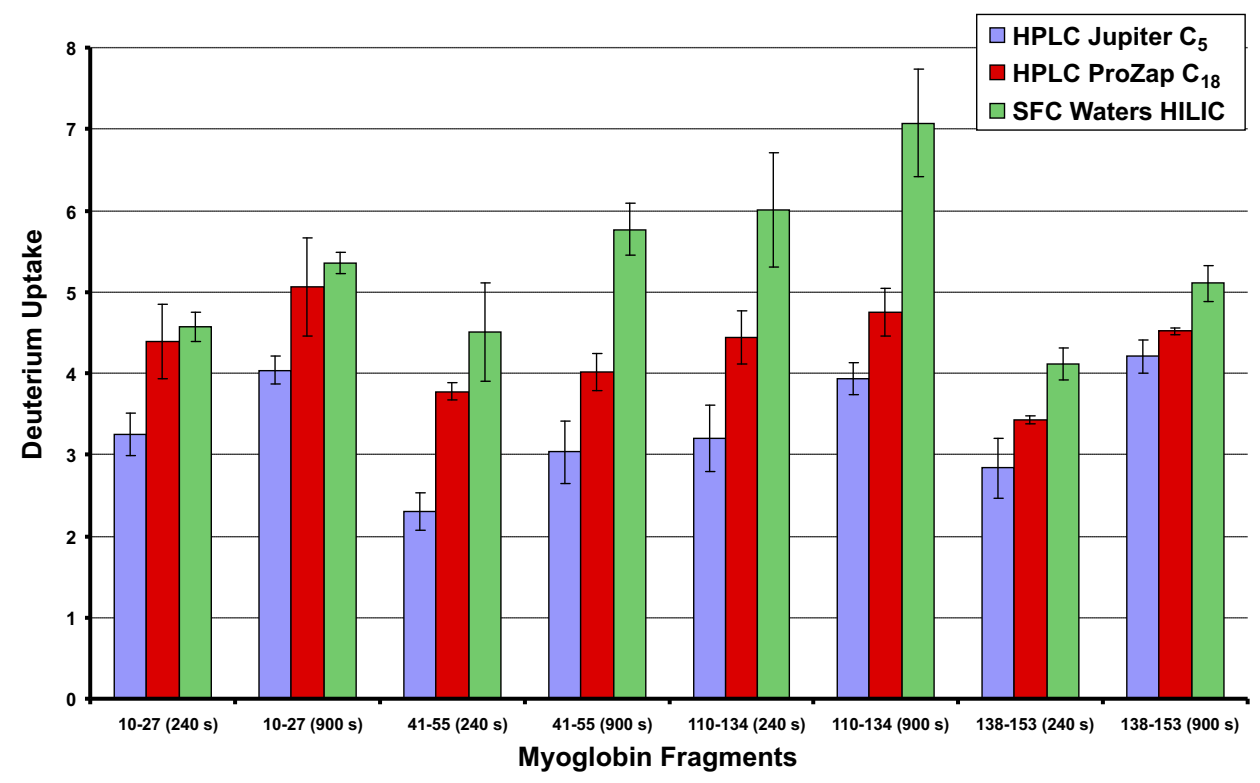

Figure 2. Deuterium uptake for myoglobin peptides after exchange for 240 and $900 \mathrm{~s}$ and separation with the Jupiter ${ }^{\mathrm{TM}} \mathrm{C}_{5}$ and ProZap ${ }^{\mathrm{TM}} \mathrm{C}_{18}$ columns with 1.5-min short gradient and SFC Waters HILIC columns. 


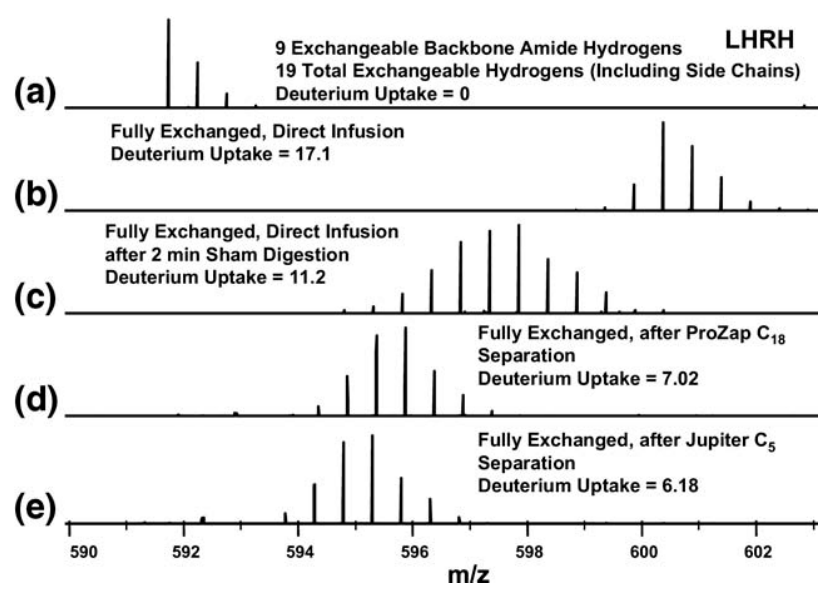

Figure 3. LHRH isotopic distribution and back exchange under various conditions. (a) LHRH natural abundance isotopic distribution from blank control (no deuterium exposure). (b) Fully exchanged LHRH (direct infusion after 56 h HDX). (c) Fully exchanged LHRH after sham digestion procedure (direct infusion after 2-min incubation in quench buffer). (d) Fully exchanged LHRH after ProZap ${ }^{\mathrm{TM}} \mathrm{C}_{18}$ LC separation. (e) Fully exchanged LHRH after Jupiter ${ }^{\mathrm{TM}} \mathrm{C}_{5}$ LC separation.

side chains were back exchanged to hydrogens (presumably arising from the $0.5 \%$ formic (protic) acid in the spray solution). In Figure 3c, close to 11 deuteriums were incorporated; thus nearly 8 deuteriums on the side chains back-exchanged and no backbone amide deuteriums were lost during the sham digestion period. The Arg D $\delta$ side-chain exchange rate is similar to that for a backbone amide hydrogen [12], but since 11 deuteriums were incorporated, clearly all of the 9 backbone amide hydrogens are retained, plus the D $\delta$ side chain of Arg and one additional side-chain hydrogen. Thus, there is no significant backbone amide hydrogen back exchange during digestion and the main back exchange occurs during the LC separation procedure.

Following the ProZap column separation, about 7 ( $78 \%$ of a possible 9 , with $0.71 \%$ error) backbone amide deuteriums remained on LHRH (Figure 3d), compared to about $6\left(69 \%\right.$, with $0.63 \%$ error) for the Jupiter $\mathrm{C}_{5}$ column separation (Figure 3e). The rapid chromatography provided by the ProZap column reduced back exchange by roughly one third (i.e., $22 \%$ versus $31 \%$ for the Jupiter $\mathrm{C}_{5}$ column).

For the Jupiter $C_{5}$ column, peptides eluted at 4.0-4.5 min (i.e., $95 \%$ organic eluent), whereas peptides eluted from ProZap $\mathrm{C}_{18}$ at $0.7-1.5 \mathrm{~min}$, i.e., highly aqueous eluent (14-64\% organic). Back exchange is thus more extensive with Jupiter $C_{5}$ than with the ProZap $C_{18}$ column. These results imply that for longer retention, more aqueous mobile phase flows past the bound peptides and thus back exchange increases. Finally, the fast RPLC column is also compatible with normal LC pumps and pressure systems (operating at $\sim 10 \mathrm{MPa}$ maximum pressure).

To assess the need for fast elution of the peptides to reduce back exchange, we tested a longer $(15 \mathrm{~min})$ gradient for both of the RPLC columns (see Supplemental Figure 1). Supplemental Figure 2 shows deuterium incorporation for myoglobin fragments with the two columns, for both short (1.5 $\mathrm{min})$ and long $(15 \mathrm{~min})$ gradients. Supplemental Figure 3 illustrates the 56-hour deuterated myoglobin and KIT deuterium uptake for identical fragments following short-gradient separation with the two columns. Supplemental figures may be found in the electronic version of this article.

\section{Conclusions}

Because of its wide inside diameter, short length, small particle size, and large pore size, the reversed-phase ProZap $\mathrm{C}_{18}$ column provides fast separation of digested peptides. Rapid elution of peptic fragments is essential to reduce back exchange and ensure accurate assignment of protein conformation. Based on fully exchanged LHRH data, we demonstrate that there is no significant backbone amide hydrogen back exchange during quench and digestion; back exchange occurs primarily during subsequent LC separation. The calculated absolute back exchange for the ProZap $\mathrm{C}_{18}$ column was about $22 \%$ and for the Jupiter $C_{5}$ was roughly $31 \%$. The reduction in back exchange is especially promising for the faster exchanging hydrogens, which are crucial to elucidate protein structure change upon ligand binding, posttranslational modifications, or mutagenesis. Rist and Mayer [13] demonstrated structural changes in enzymes in the subsecond to second timescale by use of a quenched-flow apparatus, and low back exchange is necessary and/or advantageous for those types of analysis. The fast chromatography also provides high reproducibility and high elution capacity, and has great potential for higher throughput. The column yielded a large number of overlapped fragments with high sequence coverage, for improved amino acid sequence resolution for HDX conformational studies. It is also compatible with conventional RPLC, operating at normal pressure with standard solvent pumps, and has become our standard reversedphase method for HDX analysis.

\section{Acknowledgments}

The authors thank Xiu Yu and Michael Greig at Pfizer for providing the KIT samples. The authors acknowledge Tanner Schaub and Jeremiah Tipton for their support in instrumentation and the experiments. This work was supported by NIH Grant 1R01 GM-78359, NSF Division of Materials Research through DMR-0654118, and the State of Florida.

\section{References}

1. Zhang, Z.; Smith, D. L. Determination of Amide Hydrogen Exchange by Mass Spectrometry: A New Tool for Protein Structure Elucidation. Protein Sci. 1993, 2, 522-531.

2. Engen, J. R.; Smith, D. L. Investigating Protein Structure and Dynamics by Hydrogen Exchange MS. Anal. Chem. 2001, 73, 256A-265A.

3. Lam, T. T.; Lanman, J. K.; Emmett, E. R.; Hendrickson, C. L.; Marshall, A. G.; Prevelige, P. E. Mapping of Protein:Protein Contact Surfaces by Hydrogen/Deuterium Exchange, Followed by On-line High-Performance 
Liquid chromatography-Electrospray Ionization Fourier-Transform Ion-Cyclotron-Resonance Mass Analysis. J. Chromatogr. A 2002, 982, 85-95.

4. Wales, T. E.; Engen, J. R. Hydrogen Exchange Mass Spectrometry for the Analysis of Protein Dynamics. Mass Spectrom. Rev. 2006, 25, 158-170.

5. Kipping, M.; Simmons, D. A. Protein-folding Kinetics and Mechanisms Studied by Pulsed-labeling and Mass Spectrometry. J. Mass Spectrom. 2003, 38, 271-276.

6. Emmett, M. R.; Kazazic, S.; Marshall, A. G.; Chen, W.; Shi, S. D. H.; Bolanos, B.; Greig, M. J. Supercritical Fluid Chromatography Reduction of Hydrogen/Deuterium Back Exchange in Solution-Phase Hydrogen/ Deuterium Exchange with Mass Spectrometric Analysis. Anal. Chem. 2006, 78, 7058-7060

7. Schaub, T. M.; Hendrickson, C. L.; Horning, S.; Quinn, J. P.; Senko, M. W.; Marshall, A. G. High-Performance Mass Spectrometry: Fourier Transform Ion Cyclotron Resonance at 14.5 Tesla. Anal. Chem. 2008, 80, 3985-3990.

8. Kazazic, S.; Nilsson, C. L.; Emmett, M. R.; Marshall, A. G. In Proceedings of the 5th North American FT-ICR Mass Spectrometry Conference; Key West, FL, 2005.
9. Zhang, H.-M.; Kazazic, S.; Schaub, T. M.; Tipton, J. D.; Emmett, M. R. Marshall, A. G. Enhanced Digestion Efficiency, Peptide Ionization Efficiency, and Sequence Resolution for Protein Hydrogen/Deuterium Exchange Monitored by Fourier Transform Ion Cyclotron Resonance Mass Spectrometry. Anal. Chem. Published online 11/04/2008; 2008, 80, 9034-9041.

10. Marshall, A. G.; Guan, S. Advantages of High Magnetic Field for FT-ICR Mass Spectrometry. Rapid Commun. Mass Spectrom. 1996, 10, $1819-1823$

11. Kazazic, S.; Emmett, M. R.; Blakney, G. T.; Hendrickson, C. L.; Marshall A. G. In Proceedings of the 54th American Society for Mass Spectrometry Annual Conference on Mass Spectrometry and Allied Topics; Indianapolis, IN, 2006.

12. Bai, Y.; Milne, J. S.; Mayne, L.; Englander, S. W. Primary Structure Effects on Peptide Group Hydrogen Exchange. Proteins Struct. Funct. Genet. 1993, 17, 75-86.

13. Rist, W.; Rodriguez, F. Jorgensen, T. J. D.; Mayer, M. P. Analysis of Subsecond Protein Dynamics by Amide Hydrogen Exchange and Mass Spectrometry Using a Quenched-Flow Setup. Protein Sci. 2005, 14, 626-632. 